\title{
Características e limites do mestrado profissional na área da Saúde: estudo com egressos da Fundação O swaldo Cruz
}

\author{
Characteristics and limits of Professional $\mathrm{M}$ aster in the Health area: \\ study with alumni from Oswaldo Cruz Foundation
}

Virginia Alonso H ortale ${ }^{1}$

M aria do Carmo Leal ${ }^{2}$

CarlosOtávio Fiúza M oreira ${ }^{3}$

Adriana Cavalcanti deAguiar ${ }^{3}$

\footnotetext{
${ }^{1}$ Departamento de Administração e Planejamento em Saúde, Escola Nacional de Saúde Pública, Fundação Oswaldo Cruz. Rua Leopoldo Bulhões 1.480,

Manguinhos.

21040-210 Rio de Janeiro

RJ. hortale@fiocruz.br

${ }^{2}$ Departamento de

Epidemiologia e M étodos

Quantitativosem Saúde,

Escola Nacional de Saúde

Pública, Fundação Oswaldo

Cruz.

${ }^{3}$ Fundação Oswaldo Cruz.
}

Abstract This paper discuss findings of a followup study with alumni of three graduate courses known as "professional master" oriented toward institutional innovation in management of scienceand technology, offered by Oswaldo Cruz Foundation to its employees. Thestudy analyzed alumni profile and characteristics, identified evidence of changes in their original workplaceand assessed the relationship between the courses proposals, institutional needs and alumni expectations. The paper presents limits and possibilities of this new format of graduate courses, emphasizing its evaluation as strategic for institutional development. Despite of respondents' satisfaction with the courses, this inquiry discloses institutional difficulties to incorporate some innovations, including those concerned to its institutional management.

Key words Graduate courses, Professional master, Alumni, Assessment, Follow up studies
Resumo 0 artigo discute os resultados de um estudo com egressos de três cursos de mestrado profissional na área de gestão de ciência e tecnologia em saúde oferecidos pela Fundação O swaldo Cruz a seus próprios quadros. 0 estudo analisou as características desses egressos; identificou evidências de possíveis modificações ger adas nasunidades de origem do egresso e dimensionou as relações entre a proposta dos cursos, as necessidades institucionais e as expectativas dos egressos. 0 artigo discute limites e possibilidades do mestrado profissional, ressaltando a relevância da avaliação dos processos para o desenvolvimento institucional. A pesar da satisfação dos egressos com os cursos, o estudo identificou dificuldades da instituição em incorporar algumas inovações, inclusive as referidas à gestão institucional.

Palavras-chave Pós-graduação, M estrado profissional, Egressos, Avaliação 
Introdução

Há mais de quatro décadas, o M inistério da Educação do Brasil implementou um programa de formação de pessoal de nível superior (mestrado e doutorado), tendo como referência o modelo norte-americano de formação pós-graduada. 0 mestrado foi então definido como um "grau autônomo por ser um nível da pós-graduação que proporciona maior competência científica ou profissional para aqueles que não desejam ou não podem dedicar-se à carreira científica" ${ }^{1}$.

Até 1995, a expressão "mestrado" não distinguia a natureza acadêmica ou profissional do curso, embora fosse crescente o número de instituições que qualificavam profissionais para atuar nos serviços enão somentena docência. N esse ano, a Coordenação de Aperfeiçoamento de Pessoal de Nível Superior (CAPES), órgão do Ministério da Educação que atua na expansão, re gulação e avaliação da pós-graduação stricto sensu no país, ao constatar que mudanças técnicas, econômicas e sociais demandavam profissionais com perfis de alta qualificação e produtividade, flexibilizou seu modelo, criando a modalidade profissional de mestrado, que não se destinava necessariamente à formação de docentes e pesquisadores do ensino superior. Além de fomentar os processos de qualificação acadêmico-científica - que orientam o mestrado acadêmico e 0 doutorado -, a CAPES passou a credenciar uma nova modalidade de mestrado, o profissional ou profissionalizante (M P). Este inclui em seus objetivos a inovação na orientação curricular, na composição do corpo docente e discente, no financiamento e nas parcerias institucionais ${ }^{2}$.

Em 1998, a CAPES reconheceu os cursos de (M P) com base nas seguintes premissas: (a) necessi dade da formação de profissionais pós graduados aptos a elaborar novas técnicas e processos; ( b) a relevância do caráter de terminalidade, ou seja, ênfase no aprofundamento da formação científica ou profissional conquistada na graduação, e (c) manutenção de níveis de qualidade condizentes com os padrões da pós-graduação stricto sensu e consistentes com a feição peculiar do M estrado dirigido à formação profissional ${ }^{3}$.

Essa "feição peculiar" do M P também pode ser entendida como um processo de desenvolvimento de habilidades e competências destinadas à análise de situações e resolução de problemas, que inclui a capacidade de refletir e atuar criticamente sobre seu processo de trabalho. Constituise em um dos elementos de aproximação entrea produção científica e o desenvolvimento de tec- nologias e inovação. Do ponto de vista do processo pedagógico, deveria necessariamente avançar na integração teoria-prática, autonomia de pensamento e compartilhamento de experiências. Ao discutir o significado estratégico dessa modalidade de pós-graduação, Carmen Teixeira ${ }^{4}$ comenta: A principal característica do mestrado profissionalizante reside, a nosso ver, no fato de ele ser oferecido a partir do estabelecimento de uma parceria entre instituições de serviço e instituições de ensino, levando-as ao mais alto grau de aproximação, em um processo que se tenta institucionalizar desde que se discute a necessidade de "integração ensino-serviço", forma de superação dos modelos tradicionaisdeformação na área de saúde, quais sejam, o "modo escolar", distanciado da realidade concreta, e o "modo serviço" nela mergulhado.

No entanto, essa modalidade parece ainda não ter alcançado consenso quanto à sua natureza, produtos eimpacto na sociedade brasileira: Há aqueles que dizem que essa modalidade é somenteuma atualização de cursos de especialização de natureza generalista; e aquel es que consi deram necessária a ampliação da interface com setores não acadêmicos da sociedade, formando recursos humanos para o exercício de profissões que não a dedocente-pesquisador. E na área da Saúde Pública há exemplos de mestrados profissionalizantes criados para capacitar quadros gestores de organizações quer de saúde quer de ciência e tecnologia em saúde, reconhecendo-se quea gestão éuma das variáveis centrais para a geração e aplicação dos conhecimentos produzidos visando a inovaçãa ${ }^{5}$.

As comparações entre o mestrado profissional e o mestrado acadêmico parecem inevitáveis. N este sentido, Barata reforça a hipótese de quea especificidade dessa modalidade de formação estará menos nos produtos científicos do que nas formas de articulação que ela pode favorecer entre as instituições de ensino e pesquisa e os serviços de saúde ${ }^{6}$.

Ao buscar uma definição afirmativa para o formato do MP, a CAPES ${ }^{7}$ argumenta que os cursos devem contribuir "de forma criativa para o desenvolvimento ou a indução do surgimento de campos de atividade profissional". Sua criação deve emergir, preferencialmente, da convergência de interesses entreinstituições acadêmicas eaquelas prestadoras deserviço. 0 perfil do egresso do M P fica caracterizado pela capacidade de acompanhar e incorporar os processos de inovação e produção de conhecimentos [...] e formular projetos voltados para responder aos problemas identificados em sua área de atuação e avaliar o impacto das intervenções aplicadas. Ou seja, tra- 
ta-se de profissional de alto nível, apto a compreender, realizar e avaliar pesquisas na medida em quea produção denovos conhecimentos subsidie 0 desenvolvimento organizacional. A identificação de demandas por esta formação deve partir não apenas dos indivíduos, mas também e predominantemente das instituições ${ }^{2}$.

Ainda hoje, não há respostas muito claras sobre quais áreas do conhecimento podem efetivamente contribuir para realizar inovações necessárias à sociedade a partir da formação de "mestres profissionais". A respeito do termo inovação, ver artigo de Conde e Araújo-Jorge ${ }^{8}$, em queas diver sas concepções existentes na literatura são analisadas e nos ajudam a entender seu uso na área da saúde coletiva. Já o termo formação é aqui entendido como "uma prática social específica e como uma verdadeira instituição que cumpre certas funções sociais relacionadas com a reprodução, regulação e legitimação do sistema social"9.

Nosúltimos anos, vem sefortalecendo a ideia de quea era do conhecimento leva à absorção de novos formatos organizacionais e que grande parte desse processo está associado à qualificação profissional e à qualidade dos conhecimentos produzidos.

Conforme Catani et al. ${ }^{10}$, na ótica empresarial tem prevalecido 0 entendimento de que os novos perfis profissionais eos model os de formação exi gidos atualmente pelo paradigma de produção capitalista podem ser expressos resumidamente, em dois aspectos: polivalência e flexibilidade profissionais.

Para o desenvolvimento desse "profissional multicompetente", seria necessário identificar as competências para o exercício de diferentes profissões e, consequentemente, repensar os atuais currículos da formação no estrito senso. A implementação dessa modalidade de mestrado é trabalhosa eimplica negociações difíceis que permitam flexibilizar efetivamenteas estruturas que organizam as instituições de ensino e pesquisa.

No início de 2008, segundo informações disponíveis no sítio daCAPES ${ }^{11}$, existiam 226 cursos de M $P$ recomendados nas várias áreas de conhecimento, o que representa em torno de $9 \%$ do total de 2.545 cursos de mestrado reconhecidos no país (modalidades acadêmica e profissional).

Conforme a classificação utilizada, quatro grandes áreas têm percentual de M P respondendo por mais de $10 \%$ do total de cursos. São elas: (a) outras (ensino de ciências ematemática emultidisciplinar), com 32\%; (b) ciências sociais aplicadas, com $12,9 \%$, e (c) engenharias, com $12,3 \%$. Considerando as outras grandes áreas, há maior concentração em áreas do conhecimento mais aplicadas. No entanto, na avaliação trienal 20042006, 59,7\% desses cursos foram avaliados pela CAPES com conceito 3 (regular), o que indica o atendimento a um padrão mínimo de qualidade.

Uma instituição brasileira que passou a oferecer cursos de MP é a Fundação O swaldo Cruz (Fiocruz). Trata-sedeumainstituição deciência e tecnologia destinada à pesquisa, ao ensino e ao desenvolvimento tecnológico no campo da saúde. Pertencenteao M inistério da Saúde, a Fiocruz comporta também laboratórios de referência para diagnósticos e vigilância de várias doenças e para o controle de qualidade de produtos do Sistema Ú nico deSaúde(SUS), além de produzir insumos para saúde: testes diagnósticos, imunobiológicos emedicamentos. A Fiocruz tem cerca de 4.200 servidores e mais de 5.000 terceirizados distribuídos em catorzeunidades técnico-científicas.

Em 2001, essa instituição diagnosticou a quase inexistência no país deiniciativas voltadas para a qualificação de profissionais, no campo da gestão, nas áreas de ciência e tecnologia em saúde e de informação e comunicação em saúde, o que afetava seu próprio desenvolvimento institucional. Assim, dez anos após a Portaria $n^{\circ} 47^{2}$, alguns dos dezoito programas de pós-graduação da Fiocruz, credenciados pela CAPES, passaram a oferecer seus primeiros cursos de M P. Foram titulados quase trezentos alunos para atender à necessidade de qualificação do quadro profissional da Fiocruz e de outras instituições públicas.

Esses MP foram criados com o objetivo de qualificar profissionais para atuarem como formadores e indutores de processos de mudança em seus espaços de trabalho mediante a adoção de novos conceitos e práticas, desenvolvendo produtos de alta aplicabilidade ao desenvolvimento do Sistema Ú nico de Saúde e do Sistema deCiência eTecnologia.

Este artigo discute os resultados de um estudo desenvolvido na Fiocruz, apontando alguns limites e possibilidades do mestrado profissional em instituições como essa eressaltando a relevância da avaliação dos processos instituídos para o desenvolvimento dessa modalidade de pós-graduação.

\section{O estudo}

Em 2006, foi realizado um estudo com egressos de três cursos de mestrado profissional da Fiocruz: gestão de ciência etecnologia em saúde, gestão de informação e comunicação em saúde, e 
tecnologia de imunobiológicos, que ocorreram entre 2003 e2005. Esses cursos foram credenciados pela CAPES com conceito 5 (alto nível de desempenho).

O foco do estudo foi o impacto institucional desses cursos e teve como objetivos: (a) analisar al gumas características dos egressos desses cursos; (b) identificar evidências demodificação gerada pelos cursos nas unidades de origem do egresso e (c) dimensionar as relações entrea proposta dos cursos, as necessidades institucionaise expectativas pessoais dos egressos.

Visando isolar, na medida do possível, variáveis intervenientes, empregou-se a estratégia de controle reflexivo (reflexivecontrols), na qual "sujeitos que recebem a intervenção são comparados consigo mesmos, antes e depois da intervenção" ${ }^{12}$. Esta opção foi necessária em decorrência do tempo relativamente curto en tre a conclusão dos cursos e a coleta de dados.

0 universo do estudo constou dos 66 egressos, todos funcionários da Fiocruz, e 33 chefias imediatas. Os egressos foram identificados através do Sistema de Gerenciamento Acadêmico de Alunos (SIGA), queéo sistema eletrônico de gestão do ensino da Fiocruz, de acesso restrito às secretarias acadêmicas dos institutos. N esse sistema, se encontram informações sobre a vida acadêmica eendereço eletrônico detodos os alunose egressos. As chefias foram identificadas através das cartas de recomendação emitidas por ocasião da candidatura dos seus funcionários aos cursos.

Todos receberam uma carta-convite, informando sobre os objetivos do estudo e solicitando a aceitação para participar. Dois tipos de questionários semiestruturados, um para os egressos e outro para as chefias, foram respondidos on line entre os meses de outubro e dezembro de 2006. Foi gerado um endereço eletrônico específico para esclarecimento dedúvidas econtato com a equipe da investigação, profissionais da Vicepresidência de Ensino, I nformação eComunicação da Fiocruz.

Os questionários incluíam um espaço destinado a comentários gerais, cuja análise demonstrou uma convergência de temas voltados para a política institucional e para a proposta do curso. Esse último, por não fazer parte dos objetivos originais do estudo, não será objeto de análise neste artigo.

0 número de respostas das chefias foi inexpressivo, sugerindo pouco interesse pelo processo avaliativo. Dos 39 egressos que responderam ao questionário, três foram excluídos (um deles por não pertencer aos quadros da Fiocruz e os outros dois por terem preenchido somente os dados demográficos do questionário). Um total de 36 questionários respondidos foram analisados ${ }^{13}$.

\section{Resultados}

A interpretação dos resultados levou em conta duas limitações do estudo: o momento em que a coleta dos dados foi feita, ou seja, menos de dezoito meses após a conclusão dos cursos, o que pode não ser ideal para dimensionar 0 impacto pleno da intervenção e as possíveis diferenças entre os respondentes e os que não responderam.

\section{Perfil do egresso}

Pode ser observado na Tabela 1 que há uma ligei ra predominância do sexo feminino (52,7\%) em relação ao sexo masculino (47,3\%). M ais de $80 \%$ dos egressos que participaram do estudo estão acima de quarenta anos de idade e, destes, quase a metade está acima de cinquenta anos. Todos estão há mais de dez na instituição, sugerindo uma alta correlação en tre faixa etária, tempo de serviço na instituição e a demanda pelo M P; ou seja, aqueles com mais tempo de serviço e com mais idade procuraram o curso.

A área de formação predominante dos egressos éa das ciências biológicas e saúde, seguida da área de ciências sociais aplicadas (aí incluídos administração, economia, direito, comunicação, entre outros), mostrando a diversidade de formação em uma instituição de pesquisa voltada para o setor saúde.

Implicações entre o curso

e o processo de trabalho

De acordo com a Tabela 2, dos egressos que participaram do estudo, 67\% fizeram o curso por iniciativa pessoal, enquanto apenas $31 \%$ por recomendação institucional como forma de aprimoramento profissional. Mais da metade dos egressos respondeu continuar no exercício das mesmas atividades antes do curso, podendo indicar que não houve, por parte da instituição, previsão de mudança de cargo após o aprimoramento profissional.

Pouco mais de $60 \%$ dos egressos consideraram que o curso produziu modificações substanciais no seu processo de trabalho e $72 \%$ alimentavam a expectativa de desenvolver proposta de melhoria no processo de trabalho. Além disso, $64 \%$ consideraram que o curso ampliou 
as fontes de informações sobre a área de atuação, com modificação substancial da capacidade para resolver problemas no trabalho.

Todos informaram que seus conhecimentos aumentaram, embora $47,2 \%$ tenham considerado que o curso não modificou substancialmente sua atitude como servidor. 0 curso parece ter possibilitado o acúmulo de conhecimentos dos funcionários; no entanto, 39\% dos egressos responderam que a proposta de trabalho não foi implementada, seja por falta de apoio da direção $(30 \%)$, seja por outros motivos $(70 \%)$, o que indica um baixo compromisso institucional com a recepção e implementação das mesmas, reforçando uma correlação positiva com o alto percentual da iniciativa pessoal enão de um processo indutivo institucional na realização do curso.

\section{Discussão}

A Fiocruz é uma instituição pública que realiza regularmente um congresso interno para deliberar sobre a implementação de projetos estratégicos institucionais. Um desses projetos aprovados objetivava promover a qualificação de seus quadros gestores através de cursos de mestrado profissional. Os resultados do estudo realizado

Tabela 1. D escrição sociodemográfica dos egressos dos cursos de mestrado profissional para servidores da Fiocruz. Rio de Janeiro, 2007.

\begin{tabular}{lrr}
\hline \multicolumn{1}{c}{ Indicadores } & $\mathrm{N}$ & \multicolumn{1}{c}{$\%$} \\
& 36 & $100 \%$ \\
\hline $\begin{array}{lrr}\text { Sexo } \\
\quad \text { Feminino }\end{array}$ & 19 & $52,7 \%$ \\
$\quad$ M asculino & 17 & $47,3 \%$ \\
Faixa etária & & \\
20 a 39 anos & 6 & $16,6 \%$ \\
40 a 49 anos & 13 & $36,1 \%$ \\
M ais de 50 anos & 17 & $47,3 \%$ \\
Escolaridade & & \\
$\quad$ Ciências Biológicas e Saúde & 17 & $47,2 \%$ \\
Ciências Sociais Aplicadas & 9 & $25,0 \%$ \\
Ciências H umanas e Sociais & 3 & $8,3 \%$ \\
Ciências Exatas e da Terra & 3 & $8,3 \%$ \\
$\quad$ Engenharias e Tecnologias & 4 & $11,2 \%$ \\
Tempo de serviço na instituição & & \\
$\quad$ M enos de 10 anos & 0 & $0 \%$ \\
Entre 10 e 15 anos & 19 & $52,80 \%$ \\
M ais de 15 anos & 18 & $47,20 \%$ \\
\end{tabular}

indicam dificuldades para se implementar os produtos desses cursos, em sua maioria propostas de melhoria organizacional, uso de novas metodologias referidas ao processo de trabalho e procedimentos operacionais para a produção.

É preocupante o fato de grande parte dos egressos estar acima de cinquenta anos, podendo indicar que a instituição não manteve estratégias mais explícitas de aperfeiçoamento dos seus qua-

Tabela 2. O pinião dos egressos dos cursos de mestrado profissional para servidores da Fiocruz. Rio de Janeiro, 2007.

\begin{tabular}{|c|c|c|}
\hline Indicadores & $\begin{array}{l}\mathbf{N} \\
36\end{array}$ & $\begin{array}{c}\% \\
100 \%\end{array}$ \\
\hline \multicolumn{3}{|l|}{ M otivo para cursar o mestrado profissional } \\
\hline Iniciativa pessoal & 24 & $66,7 \%$ \\
\hline $\begin{array}{l}\text { Recomendação institucional, como forma de } \\
\text { aprimoramento profissional }\end{array}$ & 11 & $30,6 \%$ \\
\hline Outros & 1 & $2,7 \%$ \\
\hline \multicolumn{3}{|l|}{ Exercício das atividades profissionais } \\
\hline Continuou exercendo as mesmas atividades & 18 & $50 \%$ \\
\hline $\begin{array}{l}\text { Passou a exercer outras atividades relacionadas } \\
\text { com a formação no curso }\end{array}$ & 12 & $33,3 \%$ \\
\hline $\begin{array}{l}\text { Passou a exercer outras atividades não } \\
\text { relacionadas à formação no curso }\end{array}$ & 6 & $16,7 \%$ \\
\hline \multicolumn{3}{|l|}{ Relação entre o curso e o processo de trabalho } \\
\hline $\begin{array}{l}\text { Produziu modificações substanciais no processo } \\
\text { de trabalho }\end{array}$ & 22 & $61,1 \%$ \\
\hline $\begin{array}{l}\text { Produziu poucas modificações no processo de } \\
\text { trabalho }\end{array}$ & 13 & $36,1 \%$ \\
\hline $\begin{array}{l}\text { N ão havia relações substanciais entre o curso e } \\
\text { as atividades desempenhadas }\end{array}$ & 1 & $2,8 \%$ \\
\hline \multicolumn{3}{|l|}{ Expectativas } \\
\hline $\begin{array}{l}\text { Desenvolver proposta de melhoria no processo } \\
\text { de trabalho }\end{array}$ & 26 & $72,2 \%$ \\
\hline Aumentar os conhecimentos na área de atuação & 9 & $25 \%$ \\
\hline Participar de um espaço de reflexão & 1 & $2,8 \%$ \\
\hline \multicolumn{3}{|l|}{ Aumento dos conhecimentos } \\
\hline $\begin{array}{l}\text { Aumentou os conhecimentos técnicos e } \\
\text { modificou substancialmente a postura como } \\
\text { servidor }\end{array}$ & 19 & $52,8 \%$ \\
\hline $\begin{array}{l}\text { Aumentou os conhecimentos técnicos, mas não } \\
\text { modificou a postura como servidor }\end{array}$ & 17 & $47,2 \%$ \\
\hline \multicolumn{3}{|l|}{$\begin{array}{l}\text { M otivo da não implementação da proposta de } \\
\text { trabalho }\end{array}$} \\
\hline Outro motivo & 14 & $(70 \%)$ \\
\hline Falta de apoio da direção & 7 & $(30 \%)$ \\
\hline \multicolumn{3}{|l|}{ Compromisso institucional com a proposta } \\
\hline Alto & 10 & $27,8 \%$ \\
\hline Médio & 12 & $33,3 \%$ \\
\hline Baixo & 14 & $38,9 \%$ \\
\hline
\end{tabular}


dros, mesmo considerando que, nos últimos cinco anos, com os concursos públicos, houve renovação de pessoal. A associação direta entre a faixa etária dos egressos e o tempo de serviço na instituição pode ser explicada tanto pela maior necessidade de aprimoramento profissional dos alunos frente às inovações tecnológicas ocorridas na organização nos últimos anos, quanto pela implementação de um recente plano de cargos e salários na instituição, no qual aqueles com título demestreincorporam no seu salário básico $52,5 \%$ de adicional de titulação (Lei $n^{\circ} 11.355$ de 19 de outubro de2006), um bom incentivo para aquele servidor com mais tempo na instituição.

Embora não se tenha encontrado estudos sobre egressos de cursos de mestrado profissional na área de gestão de ciência e tecnologia em saúde para possíveis comparações, pode-se le vantar algumas hipóteses sobre essas dificuldades a partir da análise dos comentários gerais que os respondentes fizeram sobre a política institucional.

Poder-se-ia pensar na dificuldade da instituição em incorporar, de forma descentralizada, as inovações, principalmenteaquelas referidasàárea de gestão. Um exemplo disso pode ser a baixa adesão das chefias imediatas ao estudo como reflexo do desconhecimento ou mesmo um descompromisso em relação às prioridades institucionais. $E$, como consequência, essas mesmas chefias não incentivariam seus subordinados a implementar os produtos do M P.

Um dos limites desse estudo é o elevado percentual de $41 \%$ de egressos não respondentes. Muito embora numa análise comparativa entre o grupo respondente e o outro não setenha verificado diferenças quanto à faixa etária e escolaridade, não se pode assegurar, ainda assim, a manutenção da validadeinterna da investigação. De outro lado, a ausência de resposta das chefias impediu uma análise do impacto do curso, conforme inicialmente previsto.

\section{Considerações finais}

0 desenvolvimento institucional para enfrentar os desafios do atual contexto brasileiro demanda a formação de lideranças criativas, a democratização do conhecimento esuautilização aplicadaà da gestão das organizações. Competência para interpretar informação científica e aprofundar diagnósticos institucionais que orientem a tomada de decisões são estratégicas em diversos setores, como nasáreas da saúdee da ciência etecnolo- gia. 0 curso de M P contribui para resgatar o sentido da terminalidade deste nível de pós-graduação, atualmente interpretado por muitos como mera etapa preparatória para o doutorado ${ }^{14}$.

0 estudo serviu, de um lado, para identificar fragilidades e fortal ezas da oferta de cursos dessa natureza em uma instituição como a Fiocruz. No que diz respeito aos produtos esperados, alertou a instituição para a necessidade de acionar mecanismos de fomento à implementação das propostas elaboradas. Um exemplo disso foi a criação do Programa de Incentivo à I novação no Ensino, Comunicação e Informação na Fiocruz, lançado em 2008, que promoveu um concurso dos melhores trabalhos de conclusão dos cursos de M P para terem apoio financeiro na sua implementação.

A emergência e consolidação do M $P$ também explicitam novos questionamentos sobre a pósgraduação stricto sensu e suas modalidades de avaliação. As principais premi ssas adotadas pela CAPES em relação aos M P - inovação na orientação curricular, na composição do corpo docentee discente, no financiamento e nas parcerias institucionais - deverão ser valorizadas nos processos avaliativos desse tipo de formação.

Nesse sentido, a CAPES tem buscado, nas consultas realizadas aos comitês de avaliação, subsídios para definir novos indicadores e instrumentos, mas as dificuldades inerentes a esse processo incluem uma baixa incorporação dos elementos oriundos dessa ausculta e uma baixa reflexão sobre a efeti vidade dessa modalidade de pós-graduação. 0 fato de retirar 0 item "autoavaliação" dos recentes formulários de avaliação não contribui para a disseminação da expertise inerente aos estudos avaliati vos ${ }^{15}$.

Em suma, faz-se necessário investir nas instituições para que ampliem a utilização de mecanismos de autoavaliação e reflexão sobre sua missão, currículos, estratégias de ensino-aprendizagem e avaliação discente ${ }^{16}$. Avaliar implica discutir mérito e valor, e a consolidação dos M P no país demanda apropriação ampla e formativa dos mecanismos de ensino-aprendizagem e avaliação utilizados e dos resultados obtidos e suas consequências para a sociedade.

Como decorrência do estudo realizado, foi proposto um sistema de acompanhamento de egressos na Fiocruz, o qual ainda se encontra em estágio inicial deimplantação. Acredita-se que, a médio prazo, esse sistema auxiliará na avaliação de impacto dos M P e de seus produtos, embora a chamada "cultura de avaliação" ainda tenha uma baixa penetração, apesar dos esforços institucionais já empreendidos. Com a análise contí- 
nua dos M P da Fiocruz a partir de estudos sobre egressos, como o que foi realizado, será possível gerar subsídios para outras reflexões sobre essa modalidade de pós-graduação.

\section{Colaboradores}

Os autores V Hortale, M C Leal e COF M oreira participaram, igualmente, do desenho da pesquisa, da discussão dos resultados, da redação do artigo e revisão do texto. AC Aguiar participou da discussão dos resultados, da redação e da revisão do texto.

\section{Referências}

1. Brasil. M inistério da Educação e Cultura. Parecer no 977/65 CESu-CFE, de 3 de dezembro de 1965. Define os cursos de pós-graduação. Diário Oficial da União 1966; 20 jan

2. Coordenação de Aperfeiçoamento de Pessoal do Ensino Superior. Portaria $n^{\circ} 47$, de 17 de outubro de 1995. Determina a implantação na Capes de procedimentos apropriados à recomendação, acompanhamento e avaliação de cursos de mestrado dirigidos à formação profissional. Disponível em: http://www.fnmp.org.br/documentos/portaria-no47-17-outubro-1995.pdf

3. Coordenação de Aperfeiçoamento de Pessoal do Ensino Superior. Portaria n 80 de 16 de dezembro de 1998. Dispõe sobre o reconhecimento dos mestrados profissionais e dá outras providências. Diário Oficial da U nião 1999; 11 jan.

4. Teixeira C. Significado estratégico do M estrado Profissionalizante na consolidação do campo da saúde Coletiva. In: Leal MC, Freitas CM, organizadores. Cenários possíveis: experiências e desafios do mestrado profissional na saúde coletiva. Rio de Janeiro: Fiocruz; 2006. p. 33-48.

5. Hortale VA. M estrado Profissionalizante em Saúde Pública: Um Olhar para as Experiências Internacionais. In: Leal MC, Freitas CM, organizadores. Cenários possíveis: experiências e desafios do mestrado profissional na saúde coletiva. Rio de Janeiro: Fiocruz; 2006. p. 65.

6. Barata RB. M estrado Profissionalizante: Avanços e Desafios In: Leal MC, Freitas CM, organizadores. Cenários possíveis: experiências e desafios do mestrado profissional na saúde coletiva. Rio de Janeiro: Fiocruz; 2006. p. 272.

7. Goldbaum M. Leal MC, Freitas CM, organizadores. Cenários possíveis: experiências e desafios do mestrado profissional na saúde coletiva. Rio de Janeiro: Fiocruz; 2006. p. 27. 
8. Conde MVF, Araujo-Jorge TC. Modelos e concepções de inovação: a transição de paradigmas, a reforma da C\&T brasileira e as concepções de gestores de uma instituição pública de pesquisa em saúde. Cien Saude Colet 2003; 8(3):727-741.

9. Estevão CV. Formação, Gestão, Trabalho e Cidadania Contributos Para Uma Sociologia Crítica da Formação. Educ Soc 2001; 22(77):185-206.

10. Catani AM, Oliveira JF, Dourado LF. Política educacional, mudanças no mundo do trabalho e reforma curricular dos cursos de graduação no Brasil. Educ Soc 2001; 22(75):67-83.

11. Coordenação de Aperfeiçoamento de Pessoal do Ensino Superior. [site da Internet] [acessado 2008 abr]. Disponível em: http://servicos.capes.gov.br/ projetorelacaocursos/jsp/grandeAreaD et.jsp

12. Rossi PH, Freeman HE. Evaluation: a Systematic Approach. N ewbury Park: Sage; 1993.

13. Vice-presidência de Ensino, Informação e Comunicação da Fiocruz. Coordenação Geral da Pós-graduação. Avaliação M estrado Profissional: Relatório final e recomendações. Rio de Janeiro: Fiocruz; 2007.

14. Luz MT. Prometeu Acorrentado: Análise Sociológica da Categoria Produtividade e as Condições Atuais da Vida Acadêmica. Physis 2005; 15(1):39-57.

15. Hortale VA, Moreira COF. Auto-avaliação nos programas de pós-graduação na área da saúde coletiva: características e limitações. Cien Saude Colet 2008; 13(1):223-233

16. Aguiar AC. Estratégias de Avaliação e a Transformação da Educação M édica no Brasil. Boletim da ABEM 2004; 32(5):10-11.

Artigo apresentado em 23/10/2008

Versão final apresentada em 09/02/2009 